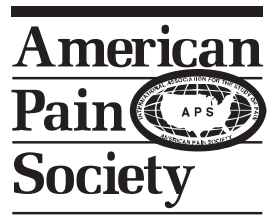

\title{
Predictors of Acute Postsurgical Pain and Anxiety Following Primary Total Hip and Knee Arthroplasty
}

*Life and Health Sciences Research Institute (ICVS), School of Health Sciences, University of Minho, Braga, Portugal. ${ }^{\dagger}$ ICVSI3B's - PT Government Associate Laboratory, Braga/Guimarães, Portugal.

${ }^{\ddagger}$ Health Psychology Group, Newcastle University, Newcastle, United Kingdom.

${ }^{\S}$ Texas Institute for Measurement, Evaluation and Statistics (TIMES) and Department of Psychology, University of Houston, Houston, Texas.

"Alto Ave Hospital Center, Orthopedics Unit, Guimarães, Portugal.

TInstitute of Health and Society, Faculty of Medical Sciences, Newcastle University, UK.

\begin{abstract}
This study aims to examine the joint role of demographic, clinical, and psychological variables as predictors of acute postsurgical pain and anxiety in patients undergoing total knee arthroplasty (TKA) and total hip arthroplasty (THA). A consecutive sample of 124 patients was assessed 24 hours before (T1) and 48 hours after (T2) surgery. Demographic, clinical, and psychological factors were assessed at $\mathrm{T} 1$ and several postsurgical pain issues, anxiety, and analgesic consumption were evaluated at $\mathbf{T} 2$. Hierarchical linear regression analyses were performed to identify predictors of acute pain and anxiety following surgery. In the final multivariate model, presurgical optimism emerged as the main significant predictor of postsurgical pain intensity. Presurgical optimism also had a significant role in the prediction of postsurgical anxiety, together with presurgical anxiety level and emotional representation of the condition leading to surgery (osteoarthritis). A significant positive correlation between postsurgical anxiety and acute pain was also confirmed. The present study enhances our understanding of predictors of acute pain and anxiety following TKA and THA by showing the relevance of psychological factors, over and above other potential clinical predictors. These findings could be used to develop targeted interventions aimed at acute postsurgical pain and anxiety management, following major joint arthroplasties.
\end{abstract}

Perspective: This article reveals the significant influence of psychological factors on the prediction of acute pain and anxiety 48 hours after primary total hip and knee arthroplasty. These results could prove useful for the design of interventions aimed at postsurgical pain and anxiety management.

(C) 2013 by the American Pain Society

Key words: Total knee arthroplasty, total hip arthroplasty, acute postsurgical pain, postsurgical anxiety, psychological predictors.

rthroplasties, specifically total knee arthroplasty (TKA) and total hip arthroplasty (THA) are among the most commonly performed surgeries world-

Received July 17, 2012; Revised December 15, 2012; Accepted December 31, 2012.

This work was supported by a Project grant (PTDC/SAU-NEU/108557/2008) and by a PhD grant (SFRH/BD/36368/2007) from the Portuguese Foundation of Science and Technology, COMPETE and FEDER.

We declare that none of the authors have any financial or other relationships that might lead to conflict of interest.

Address reprint requests to Vera Araújo-Soares, $\mathrm{PhD}$, Institute of Health \& Society, Faculty of Medical Sciences, Newcastle University, Baddiley-Clark Building, Richardson Road, NE2 4AX, Newcastle, UK. E-mail: vera. araujo-soares@ncl.ac.uk

$1526-5900 / \$ 36.00$

(C) 2013 by the American Pain Society

http://dx.doi.org/10.1016/j.jpain.2012.12.020 wide. ${ }^{52,53,58,105}$ With the aging population, a significant rise in the prevalence of knee and hip osteoarthritis is expected and consequently an increase in the number of surgical interventions such as TKA and THA, aimed at reducing pain and disability, improving functional status, and fostering quality of life. $5,41,108$ Arthroplasties are categorized as major surgeries and thus the occurrence of pain after surgery is expected, to some degree. Indeed, acute pain is the most common, anticipated, and predicted problem after surgery, ${ }^{4,100}$ being defined as an expected physiological response to a noxious chemical, thermal, or mechanical stimulus associated with surgery, trauma, or acute illness. ${ }^{13}$ Despite its predictability, it is important to implement protocols for improving postsurgical pain 
control and management. High levels of pain after surgery may have deleterious effects on individuals, both at physiological and psychological domains, ${ }^{21,26}$ hindering short- and long-term recovery, increasing length of stay, delaying ambulation and functional restoration, 9,65 and being also a key risk factor for the development of chronic postsurgical pain. ${ }^{75,94,107}$ Nevertheless, even with the most recent advances in research and the establishment of new guidelines and standards for treatment, postsurgical pain continues to be undermanaged. ${ }^{107}$

Several studies have shown that emotional distress, such as presurgical anxiety, and cognitive factors, such as pain catastrophizing, are positively correlated with postsurgical pain. ${ }^{14,48,79}$ A systematic review of predictors of postsurgical pain ${ }^{44}$ suggested that preexisting presurgical pain, anxiety, age, and type of surgery are the 4 most significant predictive factors for postsurgical pain intensity. Other potentially important but understudied determinants of acute postsurgical pain are patients' illness perceptions. The Common-Sense Self-Regulation Model ${ }^{55}$ suggests that in the context of an illness, individuals develop cognitive and emotional representations of their illness, ${ }^{43,54,78}$ which have been shown to explain outcomes in a wide range of medical conditions and in response to different treatments. ${ }^{40,67,78}$ Past studies using this theoretical perspective focused on functional activity, postsurgical adjustment, or surgical recovery, rather than on their relationship with pain outcomes. $57,60,72$

Dispositional optimism, a generalized expectation that good things will happen, ${ }^{83}$ has been identified as a significant predictor of positive outcomes in a variety of healthand disease-related conditions. 3,51,83,89,91 However, the role of optimism in postsurgical acute and chronic pain has received less attention, ${ }^{76}$ although there is some evidence of the association between optimism and surgical pain during the early recovery period. ${ }^{59}$

After surgery, pain and anxiety have been reported as being interwoven. ${ }^{61}$ Anxiety is thought to have an intensifying effect on pain experience, although it remains difficult to establish whether pain causes anxiety or whether anxiety leads to increased pain. Moreover, anxiety also has negative consequences on recovery from surgery, with some studies supporting an inverse relationship between anxiety and wound healing. ${ }^{24,45,49}$

Few studies ${ }^{85}$ have examined the impact of psychological factors on acute postsurgical pain after specific Q2] procedures such as TKR and THR. They tended to focus on demographic and clinical data ${ }^{30,71}$ and on long-term outcomes, such as chronic pain or functional status. $8,56,101$ In addition, postsurgical anxiety in the acute period after major joint arthroplasties has been targeted in only a few surgical studies, being underexplored. ${ }^{70,74}$ Since postsurgical pain and anxiety seem to be associated and influence recovery, ${ }^{14,61}$ the aims of this study were 2-fold: to examine the independent and joint contribution of demographic, clinical and psychological variables as predictors of acute postsurgical pain intensity and postsurgical anxiety, in patients undergoing TKA and THA.

\section{Methods}

This study was conducted in a central hospital in northern Portugal. Ethical approval was granted by the Hospital Research Ethics Committee and all participants were informed about the study and then read and signed the written informed consent.

\section{Participants and Procedures}

This was a prospective cohort study with time 1 (T1) and time 2 (T2) assessments performed between March 2009 and December 2010. A consecutive sample of 130 patients with osteoarthritis (see Fig 1) was enrolled (all invited participants accepted). Inclusion criteria were: 18 to 80 years old (although none were less than 40 years old); being able to understand written information (informed consent); being without psychiatric or neurologic pathology (eg, psychosis, dementia); and undergoing THA and TKA for diagnosis of coxarthrosis and gonarthrosis only (osteoarthrosis). Arthroplasties that were performed because of fall fractures were excluded, as well as hemiarthroplasties, revision, and emergency arthroplasties.

Patients were initially assessed 24 hours before (T1) and 48 hours after (T2) surgery, at the Hospital. Followup assessments were performed 4 to 6 months during the follow-up consultations. From T1 to T2 measurement points, 6 patients were withdrawn due to: canceled surgery $(n=3)$, repeated surgery/reoperation $(n=2)$, and ASA status IV along with occurrence of postsurgical delirium $(n=1)$. The final sample included the remaining 124 patients (loss to follow-up from T1 to T2 was $4.62 \%$ ) of which 60 underwent primary TKA and 64 primary THA.

\section{Measures}

Prior to the study, all instruments and study procedures were piloted in a sample of 12 patients for evaluation of their acceptability, feasibility, and comprehensibility. Those patients underwent major joint arthroplasty (6 THA and 6 TKA) at the same hospital in which the present study was conducted, and presented similar sociodemographic and clinical characteristics to the study sample. In addition, another pilot study was conducted with 20 women in the context of a study performed by our team, in the same hospital, but with hysterectomy patients. ${ }^{79-81}$

\section{Presurgical Assessment-Predictive Measures}

Upon hospital admission, 24 hours before surgery ( $T 1$ ), the following baseline questionnaires were administered, in a face-to-face interview by a trained psychologist.

\section{Socio-Demographic Questionnaire}

It included questions on age, education, residence, marital status, professional status, household and parity.

\section{Clinical Data Questionnaire}

It included questions about previous presurgical pain, its onset, duration and frequency, pain due to other 

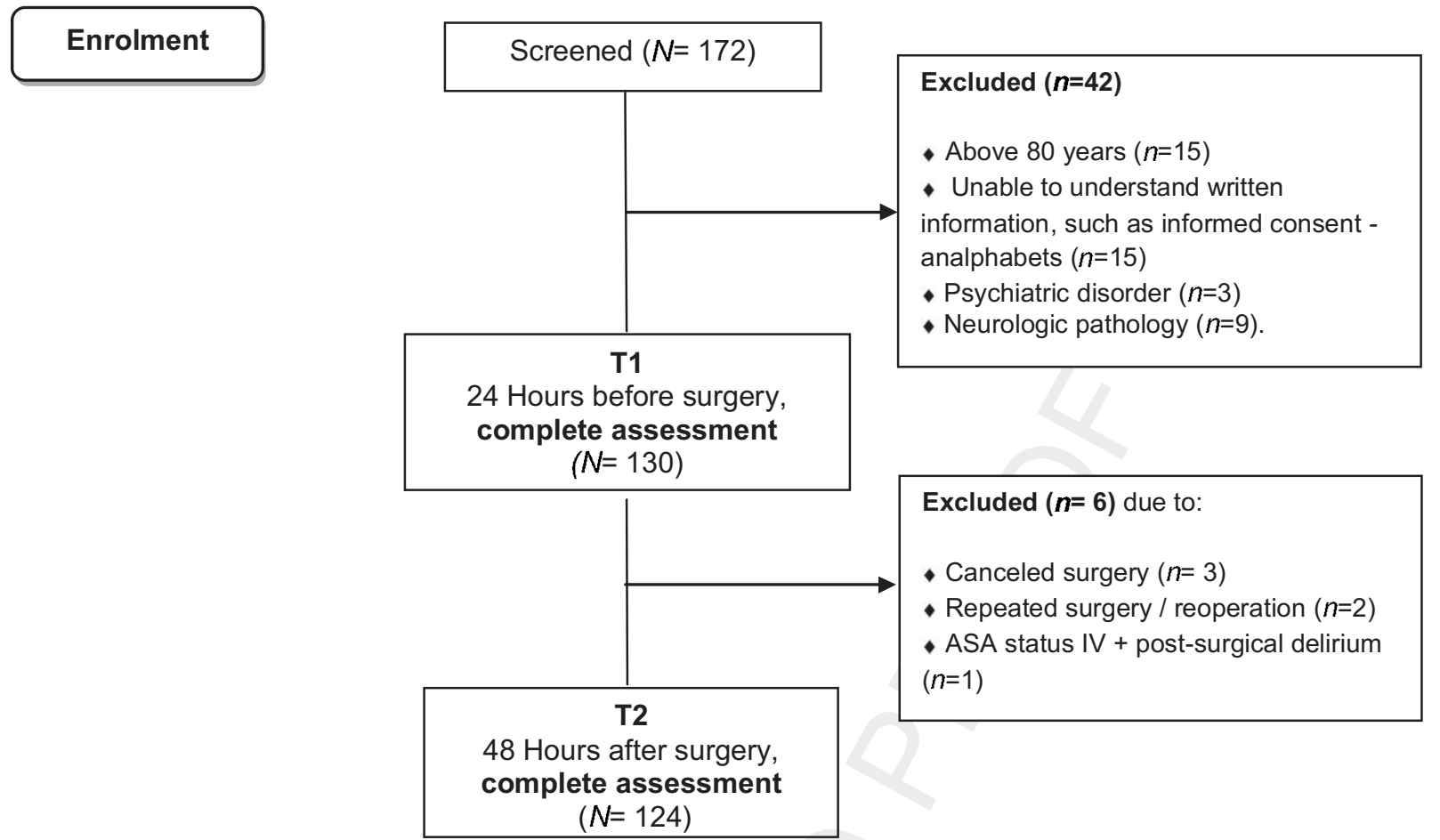

Figure 1. Flowchart of TKA and THA Patients. ${ }^{1}$

causes, pain in other joints (specifically in knees and hips), back pain, disease onset, previous surgeries, height, weight, BMI (body mass index) comorbidities, as well as the use of psychotropic drugs.

Comorbidities. The existence of presurgical comorbid conditions that could affect TKA and THA surgical outcomes was ascertained by patient interview or extracted from the medical chart. The Deyo-Charlson index ${ }^{20}$ was used, given that it is a common widely used comorbidity measure, ${ }^{41,101}$ consisting of a weighted scale of 17 comorbidities, such as hypertension, cardiac, pulmonary, renal and hepatic disease, diabetes mellitus, cancer, etc. The total number of comorbid health conditions was summed in order to yield a total score. The weighting of severity was not used in our study, but only the summative score related to the total number of comorbid conditions, as already performed elsewhere. ${ }^{46}$

\section{Brief Pain Inventory-Short Form (BPI-SF) ${ }^{22}$}

The BPI-SF measured pain intensity on an 11-point numerical rating scale (from 0 or "no pain" to 10 or "worst pain imaginable"), intake of pain analgesics, perception of analgesics relief, pain interference in daily activities (general activity, mood, walking, work, interpersonal relations, sleep, and enjoyment of life) and pain location. In this study, the internal consistency reliability ${ }^{27}$ (see Statistical Analyses) for the pain interference subscale scores was high $(\alpha=.87)$.

\section{Hospital Anxiety and Depression Scale (HADS) $^{109}$}

The HADS is comprised of 2, 7-item subscales measuring anxiety (HADS-A) and depression (HADS-D) symptomatology among patients in nonpsychiatric hospital settings. Item response format is a Likert-type scale ranging from 0 to 3 . Subscale scores vary between 0 and 21 . Higher scores represent higher levels of anxiety and depression. In the current sample, internal consistency reliability ${ }^{27}$ was adequate for both anxiety (T1: $\alpha=.79$, and T2: $\alpha=.83$ ) and depression (T1: $\alpha=.73$ ).

\section{Revised IIIness Perception Questionnaire (IPQ-R) ${ }^{68}$}

It assesses patient beliefs about the underlying condition that leads to surgery. A psychometrically short version ${ }^{98}$ was used with 7 subscales composed by 3 items each and analyzing distinct dimensions of illness perceptions: "timeline acute/chronic" $(\alpha=.97$; eg, "My illness will last for a long time"); "timeline cyclical" ( $\alpha=.56$; eg, "My symptoms come and go in cycles"); "consequences" ( $\alpha=.46$; eg, "The disease underlying surgery has major consequences on my life"); "personal control" ( $\alpha=.80$; eg, "I have the power to influence my illness"); "treatment control" ( $\alpha=.87$; eg, "Surgery can control my illness"); "illness coherence" ( $\alpha=.87$; eg, "My illness is a mystery for me"); "emotional representation" ( $\alpha=$.90; eg, "When I think about my illness I get upset"). To generate the total scale score, the sum of the item scores was divided by the number of items. Each subscale is rated on a scale of 1 to 5 , high scores revealing worst results, with the exception of personal and treatment control subscales. With the exception of the "timeline cyclical" and "consequences" subscales, which revealed low internal consistency reliability (.56 and .46), the remaining subscales presented adequate properties.

\section{Life Orientation Test-Revised (LOT-R) ${ }^{90}$}

The LOT-R evaluates the personality trait optimism via 8 items. To generate the total scale score, the sum of the 
item scores was divided by the number of items. The total score ranges from 0 to 4 with high values associated with more optimism. In the current sample, internal consistency reliability ${ }^{27}$ was very high $(\alpha=.96)$.

\section{Pain Catastrophizing Scale of the Coping Strat- egies Questionnaire-Revised Form (CSQ-R) ${ }^{84}$}

This subscale has 6 items that assess pain catastrophizing. Items were rated on a 5-point Likert scale ( $1=$ never, $2=$ almost never, $3=$ sometimes, $4=$ almost always, and $5=$ always) $)^{79-81,104}$ rather than the 7-point scale used in the original instrument, due to difficulties expressed by pilot study patients in discriminating the 7 points. To generate the total scale score, the sum of the item scores was divided by the number of items. Scale scores vary between 1 and 5, with higher scores indicating greater use of the specific coping strategy. In the current sample, the Cronbach alpha internal consistency reliability coefficient ${ }^{27}$ was .94 , indicating very good reliability.

\section{Postsurgical Assessment-Acute Pain and Anxiety}

\section{Acute Pain}

Patients were asked to rate their worst and average pain level within the first 48 hours after surgery, on an 11-point numerical rating scale (from the BPI-SF), ${ }^{22}$ already described. A composite measure was calculated, resulting from the sum and mean of worst pain level and average pain level.

\section{Anxiety}

Anxiety was measured using the anxiety subscale of the HADS, ${ }^{109}$ already described. The use of psychotropic drugs, namely the consumption of anxiolytic drugs, during the 48 hours postsurgical period, was also recorded.

\section{Postsurgical Assessment-Additional Measures}

\section{Postsurgical Pain Relief From Analgesics}

Patients were assessed on analgesic relief using a scale from the Brief Pain Inventory-Short Form (BPI-SF) ${ }^{22}$ which evaluates the perception of analgesic relief from 0 to $100 \%$.

\section{Postsurgical Pain Frequency}

This assessment was performed using the frequency subscale of the McGill Pain Questionnaire. ${ }^{63}$ Patients could define their pain either as constant (continuous, steady), intermittent (periodic, rhythmic), or brief (momentary, transient). This specific subscale was used at $\mathrm{T} 2$ because the characterization of pain that is confined to a period of 48 hours cannot be described in terms of days, weeks or months, like it was done for the assessment of presurgical pain at T1.

\section{Rescue Analgesia}

All protocols had indications for the prescription of rescue analgesics beyond the standardized analgesic protocol given moderate-to-severe acute postsurgical pain levels (Numerical Rating Scale [NRS] >3). Due to the great variability in analgesics' medications and dosages, no attempt was made to determine total equianalgesic medication dosages. Instead, it was recorded whether rescue analgesics were given to patients.

\section{Clinical Variables}

Clinical data, related to surgery, to anaesthesia and to analgesia were obtained from medical records.

After surgery, standardized postoperative nursing and physical therapy protocols were used for all patients. Patients were mobilized out of bed on the second postoperative day, and all patients had a postoperative anticoagulation protocol using LMWH (low-molecularweight heparin). After surgery, patients were given systemic prophylactic antibiotics and a prophylactic anticoagulant to decrease deep venous thrombosis risk.

Moreover, no research-related change was introduced in the standard clinical protocol. Anaesthesiologists and nurses of the acute pain team, responsible for the analgesic prescription and plans during and after the surgical procedure, were blind to their patient's participation in the study.

\section{Surgical Procedure}

From the sample of 124 patients, 60 (48\%) were submitted to TKA and $64(52 \%)$ to THA. Surgeries were performed by the team of orthopedic surgeons of the Orthopedics Unit of the above-mentioned hospital.

\section{TKA}

In the TKA surgical group, 37 patients had surgery in the right and 23 in the left knee.

For the knee, a cruciate-sacrifice prosthesis with a cobalt chromium bearing surface on an ultrahighmolecular-weight polyethylene insert surface was placed in all cases. The surgical technique in all patients was an anterior midline approach with a medial parapatellar arthrotomy. These patients all had cruciate-sacrifice TKAs with all 3 components (tibial, femoral, and patellar) cemented with a meticulous cement preparation technique. Resurfacing of the patellae was at the discretion of the surgeon. The most common technique for bone resection uses a 5 to $7^{\circ}$ (depending on body habitus) valgus femoral cut and neutral tibial cut. Additionally, a correct ligament balancing was performed and tested to achieve equal and symmetric fixation and extension gaps. Intramedullary alignment guides were used for femoral and tibial cuts. The posterior cruciate ligament was resected. Bicondylar femoral and tibial components were implanted and cemented. A polyethylene liner was inserted between the metallic femoral and tibial prostheses. When at the infirmary, a continued passive range of motion was applied to these patients, who were also instructed to weight bear as tolerated.

\section{THA}

Within this type of surgery, 34 patients had surgery in the right and 30 in the left hip. 
For hip patients, a press-fit technique was used for both components: femoral and acetabular. Supplemental screws were used to fix the cup, when necessary. Cobalt chromium on ultrahigh-molecular-weight polyethylene was the bearing surface in all cases. The surgical technique was quite similar in every case. All procedures were done through a direct anterolateral approach (Watson-Jones). The choice of surgical approach was based upon surgeon preference given the clinical scenario (ie, body habitus, severity of disease, etc). In all cases, a cementless technique was performed with a tapered stem design (to interlock in the metaphysis with no diaphyseal fixation). Moreover, proximal porous coating was used to impart stability and allow for bone ingrowth. The implant was always collarless, allowing the prosthesis to be wedged into the bony metaphysis, providing for optimal fit and bone ingrowth. In addition, the tapered design allows subsidence into a tight fit and optimizes proximal load sharing of the implant, thereby optimizing bone ingrowth and minimizing stress shielding.

For both types of surgeries, anterior-posterior (AP) hip and lateral knee x-rays were taken and reviewed after surgery and before patient was transferred to the infirmary for continued care. The x-rays were reviewed to ensure that the prosthesis was inserted properly and that alignment was correct. Compression dressings were removed the day after surgery.

\section{Anaesthetic Technique}

In all patients, the mode of anaesthesia was determined by the health care team according to the usual standard anaesthetic protocols at the hospital, with no research-related change being introduced.

The type of anaesthesia in use was classified as: 1) loco-regional alone $(n=82 / 66.1 \%)$, which could be BSA (block spinal anaesthesia) or epidural, or as; 2) locoregional (BSA or epidural) plus peripheral nerve blocks ( $n=42 / 33.9 \%)$. Among the latter, 23 (54.8\%) had a femoral nerve block, 10 (23.8\%) had a lumbar nerve block, $7(16.7 \%)$ a sciatic-femoral nerve block, and 2 $(4.8 \%)$ had a sciatic-lumbar nerve block. ASA score (physical status classification of the American Society of Anesthesiologists) was recorded, including cases of ASA grade I (9/7.3\%), II (91/73.4\%), and III (24/19.4\%).

\section{Analgesic Protocols}

All patients were assigned to a standardized analgesia protocol according to the usual standard of care at the hospital, established prior to patient transfer to the infirmary, which was determined and supervised by the acute pain unit, led by an anesthesiologist. Delivery of the analgesic protocol could be intravenous, epidural, or perineural, followed by oral analgesics on subsequent days.

The standardized intravenous protocol was composed by a continuous intravenous infusion (DIB: delivered infusion baloon) of tramadol $(600 \mathrm{mg})$, metamizol $(6 \mathrm{~g}$, and metoclopramide (60 $\mathrm{mg}$ ). The standardized epidural protocol was a continuous epidural infusion (DIB) with ropivacaine $(.1 \%)$ and fentanyl $(3 \mathrm{ug} / \mathrm{mL})$. Finally, the standardized perineural protocol included a continuous perineural infusion (DIB) with ropivacaine (.1\%). For the 3 types of protocols, Paracetamol $(1 \mathrm{~g} 6 / 6 \mathrm{~h})$ and nonsteroidal anti-inflammatory drugs (NSAIDS-ketorolac

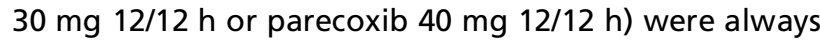
included as coadjuvant analgesics. All analgesic regimens included prokinetic treatment that was standardized to metoclopramide (10 mg i.v. $8 / 8 \mathrm{~h}$ ). All protocols had indications for the prescription of rescue analgesics beyond the standardized analgesic protocol given moderate-to severe acute postsurgical pain levels (NRS >3). ${ }^{29,42}$

\section{Statistical Analyses}

Data were analyzed using the SPSS v.19.0 software (SPSS, Inc, Chicago, IL). Internal consistency of responses to the questionnaires was assessed using Cronbach's alpha. ${ }^{27}$ The primary outcome variable in this study is "acute post-surgical pain" and the secondary outcome variable is "post-surgical anxiety," both assessed as continuous variables (pain intensity, NRS 0-10; anxiety levels, HADS 0-21). For "acute post-surgical pain," a composite measure was calculated, resulting from the sum and mean of the worst pain level and the average pain level.

Descriptive statistics were computed on sample characteristics. Furthermore, t-tests (for continuous variables) and Chi-square tests ( $\chi 2$, for nominal variables) were performed to compare demographic, clinical, and psychological measures between men and women. In addition, to determine the predictor variables to include in the regression analyses and to assess concurrent and prospective relations among study variables, Pearson correlation coefficients were calculated among continuous variables and point-biserial correlation coefficients between dichotomous and continuous variables. Multiple hierarchical regression analyses were performed to identify significant predictors of acute postsurgical pain intensity and postsurgical anxiety. The variables included in the model were the ones that showed a significant correlation with each of the dependent variables in previous bivariate associations. To control for the influence of multicollinearity, the variance inflation factor value (VIF) and the tolerance coefficients for each variable were established as being above 2 and greater than .70, respectively.

\section{Results}

\section{Sociodemographic, Clinical, and} Psychological Sample Characteristics at T1

The study sample included 41 (33.1\%) men and 83 (66.9\%) women. Table 1 displays the sample characteristics for the total sample and group differences by sex. For the total sample, mean age was 65.2 years $(S D=7.97)$; almost all patients had 4 years or less of formal education and $43.5 \%$ lived in a rural setting. With respect to clinical indicators related to pain issues, all patients complained of presurgical pain with moderate intensity and of interference on performance of daily activities (Table 1). A considerable number of patients complained of back pain, pain in other joints, and other previous pain states. These pain issues were significantly different between men and 
Table 1. Descriptive Statistics on Presurgical Sociodemographic, Clinical, and Psychological Variables for the Total Sample and Group Differences by Sex

\begin{tabular}{|c|c|c|c|c|}
\hline Patient Characteristics & Total SAMple $(N=124)$ & WOMEN $(N=83)$ & $\operatorname{MEN}(N=41)$ & $P$ \\
\hline \multicolumn{5}{|l|}{ Sociodemographic } \\
\hline Age (years[SD]) & $65.2(7.97)$ & $66.0(7.48)$ & $63.5(8.76)$ & ns \\
\hline Marital status (married) & $91(73.4 \%)$ & $54(65.1 \%)$ & $37(90.2 \%)$ & .00 \\
\hline Number of children & $3.20(2.09)$ & $3.26(2.30)$ & $3.07(1.62)$ & \\
\hline Education ( $\leq 4$ yrs) & $120(96.8 \%)$ & $81(97.6 \%)$ & $39(95.1 \%)$ & ns \\
\hline Residence (rural setting) & $54(43.5 \%)$ & $37(44.6 \%)$ & $17(41.5 \%)$ & ns \\
\hline Professional status (retired) & $93(75.6 \%)$ & $67(81.7 \%)$ & $26(63.4 \%)$ & .026 \\
\hline \multicolumn{5}{|l|}{ Clinical—general indicators } \\
\hline Disease onset (months) & $110.7(113.5)$ & $119.2(119.1)$ & $94.3(101.2)$ & ns \\
\hline $\mathrm{BMI}^{*}(\mathrm{Kg} / \mathrm{m} 2)$ & $33.7(44.3)$ & $36.0(53.8)$ & $29.1(4.39)$ & ns \\
\hline Previous surgeries (yes) & $105(85.4 \%)$ & $72(87.8 \%)$ & $33(80.5 \%)$ & ns \\
\hline Psychotropic use $†$ (yes) & $48(38.7 \%)$ & $43(51.8 \%)$ & $5(12.2 \%)$ & $<.00$ \\
\hline Comorbidities total $\ddagger$ & $2.16(1.22)$ & $2.26(1.17)$ & $1.95(1.30)$ & ns \\
\hline \multicolumn{5}{|l|}{ Clinical presurgical pain indicators } \\
\hline NRS§ (BPI): Intensity & $5.73(1.48)$ & $6.11(1.42)$ & $4.98(1.32)$ & $<.00$ \\
\hline BPI:|| Pain total interference & $28.0(12.3)$ & $31.8(11.5)$ & $20.4(10.2)$ & $<.00$ \\
\hline Pain duration $>3$ yrs & $89(73 \%)$ & $58(70.7 \%)$ & $31(77.5 \%)$ & ns \\
\hline Other prev. pain states (yes) & $81(66.4 \%)$ & $65(79.3 \%)$ & $16(40.0 \%)$ & $<.0$ \\
\hline Pain in other joints (yes) & $47(38.5 \%)$ & $38(46.3 \%)$ & $9(22.5 \%)$ & .0 \\
\hline Back pain (yes) & $60(49.2 \%)$ & $48(58.5 \%)$ & $12(30.0 \%)$ & .00 \\
\hline \multicolumn{5}{|l|}{ Psychological measures } \\
\hline HADS:** Anxiety & $5.52(4.12)$ & $6.19(4.16)$ & $4.15(3.68)$ & .00 \\
\hline HADS:** Depression & $2.38(3.13)$ & $2.80(3.29)$ & $1.54(2.64)$ & .03 \\
\hline IPQ-R:†† Timeline acut/chron. & $2.80(.94)$ & $2.88(.98)$ & $2.65(.85)$ & ns \\
\hline IPQ-R:†† Timeline cyclical & $2.97(.79)$ & $2.88(.83)$ & $3.15(.68)$ & ns \\
\hline IPQ-R:†† Treatment control & $3.98(.38)$ & $3.96(.38)$ & $4.02(.38)$ & ns \\
\hline IPQ-R: †† Illness coherence & $2.48(1.01)$ & $2.52(1.01)$ & $2.41(1.02)$ & ns \\
\hline IPQ-R:†† Emotional represent & $3.19(1.10)$ & $3.30(1.07)$ & $2.96(1.14)$ & ns \\
\hline LOT-R: $\ddagger \ddagger$ Optimism & $2.64(1.04)$ & $2.51(1.10)$ & $2.92(.83)$ & .02 \\
\hline CSQ-R: $\S$ Pain catastrophizing & $1.81(1.01)$ & $2.02(1.09)$ & $1.39(.70)$ & $<.00$ \\
\hline
\end{tabular}

NOTE. Continuous variables are presented as Mean (Standard deviation); Categorical variables are presented as $\mathrm{n}(\%)$.

*BMI.

†Psychotropic use: Consumption/Intake of anxiolytics and antidepressants.

$\ddagger$ Comorbidities total $=$ number of comorbid health conditions.

$\S N R S=0$ to 10 from BPI.

HPain Total Interference Scale 0 to 70 from BPI.

TOther previous pain states = either acute or chronic, not related to the cause of surgery, but nonetheless frequent.

**HADS.

†TIPQ-R.

$\ddagger$ †LOT-R.

$\S \S C S Q-R$.

women, with the latter presenting more pain complaints. Concerning psychological measures, on average, patients reported moderate levels of anxiety and low levels of depression symptoms, with women presenting higher scores than men (Table 1). On beliefs about the illness underlying surgery, patients tended to view their disease as chronic and cyclical (eg, perceived their disease and/or symptoms as varying over time), exhibiting a negative emotional representation of their illness, albeit reporting high expectancy of control of their problem with surgery. Moreover, patients revealed high levels of optimism and low levels of pain catastrophizing, with men showing higher scores in both factors.

\section{Surgical, Anesthetic and Postsurgical Sample Characteristics at $T 2$}

Table 2 reveals that the mean score for acute postsurgical pain was $5.26(S D=1.75)$, with women reporting higher levels of pain $(P=.001)$ and being provided more often with rescue analgesia $(P=.003)$ than men. In terms of frequency, half of the sample complained about constant, continuous, and steady pain. Regarding anxiety levels following surgery, mean score was 3.73 $(S D=3.64)$ on a scale from 0 to 21 , with no significant sex differences.

\section{Intercorrelations of Acute Postsurgical Pain (T2) and Sociodemographic, Clinical and Psychological Variables (T1, T2)}

As shown in Tables 3 and 4, acute postsurgical pain intensity and postsurgical anxiety are significantly correlated $(r=.51, P<.001)$. Table 3 shows that sex was the only demographic factor that significantly correlated with postsurgical pain intensity $\left(r_{\text {pb }}=.33, P<.001\right)$, although it did not influence postsurgical anxiety. In terms of clinical factors, pain-related variables, such as 
Table 2. Descriptive Statistics on Anesthetic, Surgical and Analgesic Variables at T2 for the Total Sample and Group Differences by Sex

\begin{tabular}{|c|c|c|c|c|}
\hline Postsurgical Data & TotaL SAMPLE $(N=124)$ & WOMEN $(N=83)$ & $\operatorname{MEN}(N=41)$ & $P$ \\
\hline Type of arthroplasty* (TKR) & $60(48.4 \%)$ & $45(75 \%)$ & $38(59.4 \%)$ & ns \\
\hline $\begin{array}{l}\text { Type of anesthesia: } \dagger \text { locoregional + } \\
\text { peripheral nerve blocks }\end{array}$ & $42(33.9 \%)$ & $32(38.6 \%)$ & $10(24.4 \%)$ & ns \\
\hline Analgesia perineural $\ddagger$ (yes) & $37(30.1 \%)$ & $28(34.1 \%)$ & $9(22.0 \%)$ & ns \\
\hline Length of hospital stay (days) & $7.16(2.88)$ & $7.14(2.15)$ & $7.22(3.98)$ & ns \\
\hline Psychotropic use $\S$ (yes) & $53(42.7 \%)$ & $43(51.8 \%)$ & $10(24.4 \%)$ & .004 \\
\hline HADS:|| Anxiety & $3.73(3.64)$ & $4.07(3.66)$ & $3.02(3.55)$ & ns \\
\hline \multicolumn{5}{|l|}{ Clinical pain \& analgesic indicators } \\
\hline NRS $\uparrow(B P I):$ Intensity & $5.26(1.75)$ & $5.67(1.47)$ & $4.44(1.98)$ & .001 \\
\hline Frequency: ${ }^{* *}$ Constant & $62(50 \%)$ & $45(54.2 \%)$ & $17(41.5 \%)$ & ns \\
\hline Rescue analgesics (yes) & $50(40.3 \%)$ & $41(49.4 \%)$ & $9(22.0 \%)$ & .003 \\
\hline$\%$ Relief from analgesics (0-100\%) & $90.5(20.0)$ & $91.9(16.8)$ & $87.50(25.4)$ & ns \\
\hline
\end{tabular}

NOTE. Continuous variables are presented as Mean (Standard deviation); Categorical variables are presented as $n$ (\%); T2, 48 hours after surgery.

*Type of arthroplasty: TKR versus THR.

$\dagger$ Type of anesthesia: Anesthesia locoregional alone: BSA or epidural versus anesthesia locoregional (BSA or epidural) + peripheral nerve blocks.

$\ddagger$ Analgesia perineural versus intravenous and epidural analgesia.

§Psychotropic use: Consumption/intake of anxiolytics and antidepressants.

IIHADS.

INRS $(\mathrm{BPI})=0$ to 10 from BPI.

**Pain Frequency: constant pain versus intermittent or brief pain, assessed via frequency subscale of McGill Pain Questionnaire.

presurgical pain interference $(r=.37, P<.001)$, pain due to other causes $\left(\mathrm{r}_{\mathrm{pb}}=.34, P<.001\right)$, and presurgical pain intensity $(r=.26, P<.01)$ were significantly correlated with acute postsurgical pain intensity, although correlation sizes were low. Regarding postsurgical anxiety, it is significantly correlated with presurgical pain interference $(r=.37, P<.001)$, presenting lower but also significant associations with pain due to other causes $\left(r_{p b}=.22, P<.05\right)$, back pain $\left(r_{\mathrm{pb}}=.28, P<.01\right)$ and total number of comorbidities $(r=.19, P<.05)$. None of the other factors revealed significant relationship with postsurgical anxiety.

Regarding psychological measures (Table 4), postsurgical pain was inversely correlated with optimism $(r=-.37$, $P<.001)$, and positively correlated with pain catastroph- izing $(r=.35, P<.001)$, and emotional representation of the condition that led to surgery (osteoarthritis; $r=.34, P$ $<.001)$, approaching the moderate range. Other psychological variables, such as presurgical anxiety and depression, also correlated significantly with postsurgical pain but in the low range. In contrast, postsurgical anxiety presented a moderate correlation with postsurgical pain $(r=.51, P<.001)$. Presurgical anxiety and presurgical depression were significant correlates of postsurgical anxiety $(r=.54, P<.001 ; r=.40$, $P<.001$, respectively). Similarly to acute postsurgical pain, optimism $(r=-.39, P<.001)$, pain catastrophizing $(r=.33, P<.001)$, and emotional representation of the condition leading to surgery $(r=.52, P<.001)$ were

Table 3. Pearson and Point-Biserial Correlation Coefficients Between Demographic and Clinical Variables (T1) and Acute Postsurgical Pain, and Postsurgical Anxiety (T2)

\begin{tabular}{|c|c|c|c|c|c|c|c|c|c|c|c|c|c|}
\hline & 1 & 2 & 3 & 4 & 5 & 6 & 7 & 8 & 9 & 10 & 11 & 12 & 13 \\
\hline 1. Acute Pain T2 & - & & & & & & & & & & & & \\
\hline 2. HADS: Anxiety T2 & $.51 * * *$ & - & & & & & & & & & & & \\
\hline 3. Age & .13 & .01 & - & & & & & & & & & & \\
\hline 4. Sex & $.33^{* * *}$ & .14 & .15 & - & & & & & & & & & \\
\hline 5. Presurgical pain intensity & $.26 * *$ & .16 & .02 & $.36 * * *$ & - & & & & & & & & \\
\hline 6. Presurgical pain interfere. & $.37 * * *$ & $.37 * * *$ & .02 & $.44^{\star \star \star}$ & $.55^{\star \star \star}$ & - & & & & & & & \\
\hline 7. Other previous pain states & $.34 * * *$ & $.22 *$ & .05 & $.39 * * *$ & $.20^{*}$ & $.38 * * *$ & - & & & & & & \\
\hline 8. Pain in other joints & $.23^{*}$ & .13 & .17 & $.23^{*}$ & .11 & $.24 * *$ & $.56 * * *$ & - & & & & & \\
\hline 9. Back pain & $.20 *$ & $.28 * *$ & .06 & $.27 * \star$ & .16 & $.29 * *$ & $.63 * * *$ & $.30 * *$ & - & & & & \\
\hline 10. BMI & -.04 & -.02 & .00 & .07 & .09 & .05 & .09 & .14 & .11 & - & & & \\
\hline 11. Comorbidities total & $.19 *$ & $.19^{*}$ & $.21 *$ & .12 & .10 & .17 & $.52 * * *$ & $.62^{* * *}$ & $.58 * * *$ & .17 & - & & \\
\hline 12. Previous surgeries & .16 & -.00 & -.07 & .10 & .15 & .11 & $.24 * *$ & .14 & .17 & .04 & .13 & - & \\
\hline 13. Type of anesthesia & $-.23^{*}$ & -.11 & .09 & -.14 & -.02 & -.08 & $-.26 * \star$ & $-.31 * \star \star$ & -.08 & .05 & -.18 & -.15 & - \\
\hline
\end{tabular}

NOTE. T1, 24 hours before surgery; T2, 48 hours after surgery; Sex, $0=$ men and 1 = women; Acute Pain and Presurgical pain T1, NRS score from BPI-SF. [Q6] Inventory-Short Form; Presurgical pain interference from BPI-SF: Brief Pain Inventory; BMI = body mass index; Type of anesthesia, $0=$ Anesthesia loco-regional. $($ BSA or epidural) + peripheral nerve blocks and $1=$ Anesthesia loco-regional alone: BSA or epidural.

$\star P<.05$

$* * P<.01$.

$* * * P<.001$ 
Table 4. Pearson Correlation Coefficients Between Baseline Psychological Variables (T1) and Acute Postsurgical Pain and Postsurgical Anxiety (T2)

\begin{tabular}{|c|c|c|c|c|c|c|c|c|c|c|c|}
\hline & 1 & 2 & 3 & 4 & 5 & 6 & 7 & 8 & 9 & 10 & 11 \\
\hline 1. Acute Pain T2 & - & & & & & & & & & & \\
\hline 2. HADS: Anxiety T1 & $.22 *$ & - & & & & & & & & & \\
\hline 3. HADS: Depression & $.27 * *$ & $.51 * * *$ & - & & & & & & & & \\
\hline 4. IPQ-R: Timeline acute/chronic & .08 & $.23^{*}$ & $.32 * * *$ & - & & & & & & & \\
\hline 5. IPQ-R: Personal control & -.04 & .15 & -.03 & $.23 * *$ & - & & & & & & \\
\hline 6. IPQ-R: Treatment control & -.15 & $-.28^{* *}$ & $-.37 * * *$ & $-.27^{* *}$ & -.07 & - & & & & & \\
\hline 7. IPQ-R: Illness coherence & .05 & -.05 & $.18^{*}$ & .02 & -.07 & -.10 & - & & & & \\
\hline 8. IPQ-R: Emotional representation & $.34 * * *$ & $.58 * \star \star$ & $.40 * \star \star$ & .17 & .01 & -.16 & .03 & - & & & \\
\hline 9. LOT-R: Optimism & $-.37 * * *$ & $-.40 * * *$ & $-.50 * * \star$ & $-.26 * *$ & .02 & $.33 * * \star$ & -.05 & $-.31 * * *$ & - & & \\
\hline 10. CSQ-R: Pain catastrophizing & $.35 * * *$ & $.54 * * *$ & $.51 * * *$ & .15 & -.07 & $-.22 *$ & -.00 & $.55 * * *$ & $-.42 * * *$ & - & \\
\hline 11. HADS: Anxiety T2 & $.51 * * *$ & $.54 * * *$ & $.40 * * *$ & $.23^{* *}$ & -.08 & -.21 & -.02 & $.52 * * *$ & $-.39 * * *$ & $.33 * * *$ & - \\
\hline
\end{tabular}

[Q7] NOTE. T1, 24 hours before surgery; T2, 48 hours after surgery. Acute Pain.

$* P<.05$

$* * P<.01$

$* * * P<.001$

significant correlates of postsurgical anxiety. These results were used to select the set of sociodemographic, clinical, and psychological predictors to include in the regression models.

\section{Predicting Postsurgical Pain Intensity and Anxiety Levels After Hip and Knee Arthroplasties}

To determine the predictors of postsurgical pain intensity and anxiety, separate multiple hierarchical regression analyses were conducted (Tables 5 and 6). Yet, this study sought to find a unique model that could predict both outcomes. In each regression, sex was included in the first step due to its significance in bivariate associations and in previous studies. ${ }^{33,47,73}$ In the following step, the presurgical score of the dependent variable (presurgical pain intensity or presurgical anxiety) was added, along with pain due to other causes. For postsurgical anxiety prediction, pain due to other causes and presurgical anxiety were split into individual steps, given their different nature. Concerning the postsurgical pain model, initially presurgical pain intensity and interference were entered along with pain due to other causes (absent, present) in the second step. However, due to problems of multicollinearity (VIF $>2$, Tolerance $<.70$ ), presurgical pain interference was excluded from this step. Other previous pain variables could also have been entered, such as pain in other joints or back pain; however, both
940

941

942

943

944

945

946

947

948

949

950

951

952

953

954

955

956

957

958

959

960

961

962

963

964

965

966

967

968

969

970

971

972

973

974

975

976

977

978

979

980

981

982

983

984

985

986

987

988

989

990

991

992

993

994

995

996

997

998

999

1000

1001

1002

${ }^{*}$ Dichotomous variable: $0=$ men and $1=$ women

$\dagger$ Continuous variable: NRS 0 to 10 from BPI-SF.

$\ddagger$ Dichotomous variable: $0=$ no, $1=$ yes.

$\S$ Continuous variable: LOT-R.

ICContinuous variable, IPQ-R (emotional representation subscale). 
Table 6. Hierarchical Multiple Regression Results for Presurgical Predictors of Postsurgical Anxiety 48 Hours After Hip and Knee Arthroplasties ( $N=124)$

\begin{tabular}{|c|c|c|c|c|c|}
\hline VARIABLES & $T$ & $\beta$ & $R^{2}$ & $\Delta R^{2}$ & $\Delta F$ \\
\hline Step 1 & & & & .013 & 1.546 \\
\hline Sex* & 1.243 & 114 & & & \\
\hline Step 2 & & & & .028 & 3.472 \\
\hline Other previous pain states $\dagger$ & 1.863 & .183 & & & \\
\hline Step 3 & & & & .277 & $47.172 * * *$ \\
\hline Presurgical anxiety & $6.868 * * *$ & .558 & & & \\
\hline Final model & & & .401 & & \\
\hline \multicolumn{6}{|l|}{ Step 1} \\
\hline Sex* & -.716 & -.057 & & & \\
\hline \multicolumn{6}{|l|}{ Step 2} \\
\hline Other previous pain states $\dagger$ & -.050 & -.004 & & & \\
\hline Step 3 & & & & & \\
\hline Presurgical anxietył & $3.833 * \star \star$ & .358 & & & \\
\hline Step 4 & & & & .082 & $7.801 * * *$ \\
\hline Optimism§ & $-2.579 *$ & -.211 & & & \\
\hline Emotional representation\| & $2.671 * *$ & .238 & & & \\
\hline
\end{tabular}

NOTE. T1, 24 hours before surgery; $T 2,48$ hours after surgery.

${ }^{\star} P \leq .05$

$* * P \leq .01$.

$* * * P \leq .001$.

*Dichotomous variable: $0=$ men, $1=$ women

†Dichotomous variable: $0=$ no, $1=$ yes.

$\ddagger$ Continuous variable, HADS (anxiety subscale).

$\S$ Continuous variable, LOT-R.

||Continuous variable, IPQ-R- (emotional representation subscale).

were highly correlated with pain due to other causes. Therefore, pain due to other causes was chosen and kept in the model instead of the other 2 pain variables, since it seems to fully incorporate the other 2 measures. In terms of psychological variables expected to have the largest impact on postsurgical pain and anxiety, the selected ones were those with highest correlations with the outcome (Table 4). Therefore, optimism and emotional representation of the condition leading to surgery (osteoarthritis) were entered together in the last step. Pain catastrophizing had to be excluded due to collinearity issues with these 2 psychological predictors.

Table 5 shows that sex was a significant predictor $(\beta=.34, P<.001)$, explaining $11.4 \%$ of the variance in postsurgical pain. When adding presurgical pain intensity and pain due to other causes (step 2 ), only the latter emerged as a significant predictor $(\beta=.24, P=.01)$. Both variables in step 2 accounted for an additional $7.1 \%$ of the variance. Psychological variables were entered in the final step and explained an additional $9.3 \%$ of the variance in postsurgical pain intensity, contributing significantly to the $27.8 \%$ variance explained by the final model. However, optimism was the only significant predictor for step 3 , emerging as the only significant predictor in the final model ( $\beta=-.24, P=.008$ ). Sex and pain due to other causes ceased to be significant, although sex presented a trend toward significance $(\beta=.17, P=.061)$, as well as emotional representation $(\beta=.17, P=.069)$.

The results of the hierarchical regression for the prediction of postsurgical anxiety are presented in Table 6. In this model, sex and pain due to other causes were entered in the first and second steps. Neither predictor reached statistical significance. Entered in the third step, presurgical anxiety proved to be a very strong and significant predictor $(\beta=.56, P<.001)$, explaining an additional $27.7 \%$ of the variance in postsurgical anxiety. In the final step, optimism and emotional representation were added, both emerging as significant predictors ( $\beta=-.21, P=.011 ; \beta=.24, P=.009$, respectively) and adding $8.2 \%$ to the explained variance. In the final model, presurgical anxiety remained a significant predictor $(\beta=.36, P<.001)$ and this final model explained $4.1 \%$ of the total variance in postsurgical anxiety. Although presurgical anxiety levels $(M=6.52 ; S D=4.11)$ dropped significantly after surgery $(M=3.73 ; S D=3.64$; $\mathrm{t}=5.349 ; P<.001)$, they still remained the best predictor of anxiety after surgery.

\section{Discussion}

This study reveals the significant influence of psychological factors in the prediction of acute pain and anxiety 48 hours after primary THA and TKA. Presurgical optimism emerged as the most significant predictor of acute postsurgical pain intensity, although history of pain was also a significant predictor. For postsurgical anxiety, presurgical anxiety was the most important predictor, although other psychological variables, such as presurgical optimism and illness emotional representation, were also important predictors. Psychological variables emerged as predictors over and above clinical. These results could prove useful for the design of interventions targeting postsurgical pain and anxiety management. 


\section{Prevalence of Acute Postsurgical Pain and Anxiety Following TKA and THA}

Despite being medicated with analgesics delivered through continuous infusions (DIBs), patients still reported moderate levels of postsurgical pain intensity 48 hours after TKA and THA ( $M=5.26$ in 10). This reflects what has been reported in other studies, ${ }^{44,62}$ in which orthopedic surgeries emerged as 1 of the most painful procedures. Accordingly, $40.3 \%$ of this sample had to be administrated rescue analgesia.

The association between acute postsurgical pain and postsurgical anxiety draws attention to the complex psychological and biological interplay of these 2 alarm systems. ${ }^{102}$ During the postsurgical period, changes in anxiety seem significantly related with changes in pain. ${ }^{14}$ This relationship is also presented in diverse chronic pain conditions, even after adjusting for potential confounding variables. ${ }^{61}$ In this sample, there were moderate levels of presurgical anxiety that dropped after surgery. Despite this, there was a moderate positive correlation between postsurgical pain and anxiety, which confirms previous findings and supports their association.

\section{Predicting Acute Postsurgical Pain and Anxiety Following TKA and THA}

Sex and pain due to other causes were initially significant predictors of acute postsurgical pain, in line with other studies. ${ }^{16,33,47,73,79}$ In the final model, only presurgical optimism emerged as significant. Sex and emotional illness representations did retain marginal significance, needing to be revisited in further studies. However, these results suggest that in patients undergoing TKA or THA, presurgical optimism seems to be the best indicator of the likelihood of patients reporting heightened pain 48 hours after surgery, irrespective of the patient's previous pain experience.

Dispositional optimism ${ }^{76}$ has been identified as a significant predictor of positive outcomes in a variety of healthand disease-related conditions. ${ }^{3,17,51,83,89,91}$ Moreover, the association between optimism and low levels of pain was shown in adult patients with several pathologies. 2,32,96,106 Optimism is associated with augmented pain tolerance and diminished pain sensitivity, ${ }^{25,35}$ being a significant predictor of placebo analgesia. ${ }^{34,66}$ The advantages of higher optimism in its relation to surgical pain were found only during the early recovery period, ${ }^{59}$ as it did not predict pain 6 months after various surgical procedures, ${ }^{77}$ although it influenced quality of life and surgical recovery. $7,18,77,83,91$

There are possible explanations for the influence of optimism on short-term surgical pain. Since arthroplasties are the last and only solution for certain impairments, it is plausible that those patients who are optimistic will confront surgery and the acute postsurgical period more positively. This could affect acute pain perception, probably because they would be less attentive to pain stimuli, ${ }^{1,35}$ focusing on their hopeful medium-term life improvements and being more keen to bear pain and other negative outcomes in the short-term period after surgery. This perspective could also lead optimistic pa- tients to engage in more adaptive coping strategies, such as positive reinterpretation, acceptance, and reliance on problem-focused coping. ${ }^{88,92}$ In our study, optimism revealed a significant negative correlation with pain catastrophizing, a maladaptive pain-coping strategy. As the choice of coping strategies appears to be the mediation mechanism through which optimism is related to less pain and distress and to better health outcomes, ${ }^{90,92}$ future investigations should focus on further assessing the relation among optimism, coping strategies, postsurgical anxiety, and pain. Finally, optimism could influence acute postsurgical pain experience through its impact on the immune system. The pro-inflammatory cytokine IL-6 has a central role in inflammation and immunity, showing increased systemic levels during physical and psychological stress. ${ }^{39,64}$ Optimistic appraisals have shown to influence the biological stress response, by counteracting acute increases in IL-6 responses. ${ }^{11,25}$ Contrarily, an association between low optimism, high pain sensitivity, and exaggerated inflammatory response to stress has been associated with high levels of IL-6. ${ }^{25}$

Given that after surgery, pain and anxiety have been reported as being interrelated, ${ }^{61}$ we examined whether postsurgical anxiety could be predicted using a model similar to the one predicting postsurgical pain. As expected, findings revealed that presurgical anxiety is the most important predictor of postsurgical anxiety experience, in line with other studies. ${ }^{13,15,28,69}$ Presurgical optimism also predicted postsurgical anxiety. The current study is the first examining this specific relationship using the LOT-R questionnaire. Although using a different measure-The Future Self-Perception Questionnaire-that evaluates hopelessness and an optimistic view of the future, another study found that a negative presurgical perception of the future was an independent risk factor for postsurgical anxiety. ${ }^{15}$ Indeed, it has been shown that high dispositional optimism is associated with an adaptive response to health-related stress, $^{34}$ being a determinant of psychological well-being. ${ }^{19,93}$ Dispositional optimism, and the ability of optimists to appraise a stressor in a more positive frame, may buffer the impact of stress on psychological states and on biological processes, ${ }^{11,23}$ which might explain why optimistic patients experience less anxiety after arthroplasty.

Another interesting finding is that in face of the prospect of undergoing an arthroplasty, patients who have a more negative emotional representation of their surgical disease (osteoarthritis), are also more likely to experience anxiety 48 hours after surgery. Patients' beliefs about whether their illness has an emotional impact, such as feeling depressed, angry, or upset, appear to influence immediate surgical outcomes. Other studies demonstrate that the emotional representation of a disease relates with health outcomes. $6,57,87$

\section{Limitations of the Study}

This is a single-site and single-country study, which limits its external validity. Therefore, the generalization 
of the conclusions to populations in other countries should be considered with caution. Additionally, the low educational level of the sample may also impact the external validity, in that different results may emerge in more educated patient populations undergoing TKA or THA. Previous research has indicated that more educated patients tend to experience less pain ${ }^{31,103}$ and be more optimistic ${ }^{37}$ and less anxious before surgery. ${ }^{50}$ Therefore, the impact of educational level on the relationships tested in this study deserves further study.

Concerning the internal validity, a potential limitation could be associated with the researcher's gender (female). Previous studies reported sociocultural influences on pain perception, which might be affected by gender "norms" and gender roles. ${ }^{82}$ Males seem to report less pain and higher thresholds when tested by a female examiner. ${ }^{36}$

Finally, we are aware that the models tested to predict postsurgical pain and anxiety are limited and that other predictors need to be explored in order to increase our understanding. Nevertheless, the results show that a considerable amount of variance could be predicted by the models tested and that psychological predictors accounted for a significant amount of variance in postsurgical pain.

\section{Implications for Clinical Practice}

Our study identified psychological factors influencing postsurgical pain that are amenable to change via appropriate presurgical psychological interventions: presurgical anxiety, optimism, and emotional representation. This knowledge has the potential to guide prevention and treatment strategies.

To address presurgical anxiety, brief cognitivebehavior intervention techniques (such as brief relaxation, imagery, and positive coping self-statements) are the gold standard, ${ }^{10,38,61,86}$ as well as reassurance and information provision. ${ }^{10,97,99}$

Concerning optimism, pain patients can benefit from interventions to increase optimism, albeit temporarily, through short visualization interventions. ${ }^{76}$ Cognitive therapy can also increase optimism levels. ${ }^{95}$ Present findings suggest that arthroplasty patients could benefit from such presurgical preventive interventions, target-

\section{References}

1. Affleck G, Tennen H, Apter A: Optimism, pessimism, and daily life with chronic illness, in Chang EC (ed): Optimism And Pessimism: Implications for Theory, Research, and Practice. Washington D.C., American Psychological Association, 2001, pp 147-168

2. Allison PJ, Guichard C, Gilain L: A prospective investigation of dispositional optimism as a predictor of healthrelated quality of life in head and neck cancer patients. Qual Life Res 9:951-960, 2000

3. Andersson G: The benefits of optimism: a meta-analytic review of the life orientation test. Pers Individ Dif 21: 719-725, 1996 ing the promotion of optimism associated with surgery outcomes, to reduce pain and anxiety following surgery.

The present study also suggests that interventions based on addressing the presurgical negative representations generated by the illness may support patients to cope more adequately with surgery. Other studies have found that challenging dysfunctional illness representations is effective in reducing disability and improving functioning. ${ }^{18,78}$ Patients should be assisted in learning and applying more effective coping skills, namely through cognitive-behavioral techniques, to change the cognitive representations regarding the negative emotional impact of their illness. ${ }^{43}$ This specific intervention, aimed at restructuring illness cognitions, could be implemented along with interventions aimed at improving optimism. The present study also emphasizes the relationship between postsurgical pain and anxiety, suggesting that they share common psychological predictors that could be targeted in intervention planning. For instance, interventions targeting optimism, a predictor of both outcomes, could benefit both postsurgical pain and anxiety, favoring a positive recovery. Given that presurgical anxiety is a strong predictor of postsurgical anxiety, intervention on presurgical anxiety would benefit postsurgical anxiety and most likely postsurgical pain. Additionally, in the acute postsurgical period, interventions targeting the decrease of anxiety may consequently reduce pain in patients whose pain is amplified by anxiety. ${ }^{102}$

Data from the present study, focusing on the shortterm postsurgical period, confirmed the influence of psychological factors on acute pain and anxiety following major joint arthroplasties. By identifying at risk patients, more appropriate psychological interventions and better postsurgical surveillance can be implemented.

\section{Uncited Reference}

12.

\section{Acknowledgments}

We thank all patients who agreed to participate in this study.

4. Apfelbaum JL, Chen C, Mehta SS, Gan TJ: Postoperative pain experience: results from a national survey suggest postoperative pain continues to be undermanaged. Anesth Analg 97:534-540, 2003

5. Bachmeier CJM, March LM, Cross MJ, Lapsley HM, Tribe K, Courtenay BG, Brooks PM, Arthritis Cost, Outcome Project Group: A comparison of outcomes in osteoarthritis patients undergoing total hip and knee replacement surgery. Osteoarthritis Cartilage 9:137-146, 2001

6. Botha-Scheepers S, Riyazi N, Kroon HM, Scharloo M, Houwing-Duistermaat JJ, Slagboom E, Rosendaal FR, Breedveld FC, Kloppenburg M: Activity limitations in the lower extremities in patients with osteoarthritis: the modifying effects of illness perceptions and mental health. Osteoarthritis Cartilage 14:1104-1110, 2006 
12 The Journal of Pain

1381

1382

1383

1384

1385

1386

1387

1388

1389

1390

1391

1392

1393

1394

1395

1396

1397

1398

1399

1400

1401

1402

1403

1404

1405

1406

1407

1408

1409

1410

1411

1412

1413

1414

1415

1416

1417

1418

1419

1420

1421

1422

1423

1424

1425

1426

1427

1428

1429

1430

1431

1432

1433

1434

1435

1436

1437

1438

1439

1440

1441

1442

1443
7. Bowley D, Butler M, Shaw S, Kingsnorth A: Dispositional pessimism predicts delayed return to normal activities after inguinal hernia operation. Surgery 133:141-146, 2003

8. Brander VA, Gondek S, Martin E, Stulberg SD: Pain and depression influence outcome 5 years after knee replacement surgery. Clin Orthop Relat Res 464:21-26, 2007

9. Brander VA, Stulberg SD, Adams AD, Harden RN, BruehIS, Stanos SP, Houle T: Predicting total knee replacement pain a prospective, observational study. Clin Orthop Relat Res 416:27-36, 2003

10. Bruehl S, Chung OY: Psychological Interventions for Acute Pain, in Hadjistavropoulos T, Craig KD (eds): Pain: Psychological Perspectives. Mahwah, NJ, Lawrence Erlbaum Associates, 2004, pp 245-269

11. Brydon L, Walker C, Wawrzyniak AJ, Chart H, Steptoe A: Dispositional optimism and stress-induced changes in immunity and negative mood. Brain Behav Immun 23:810-816, 2009

12. Carr E, Brockbank K, Allen S, Strike P: Patterns and frequency of anxiety in women undergoing gynaecological surgery. J Clin Nurs 15:341-352, 2006

13. Carr DB, Goudas LC: Acute pain. Lancet 353:2051-2058, 1999

14. Carr ECJ, Thomas VN, Wilson-Barnet J: Patient experiences of anxiety, depression and acute pain after surgery: A longitudinal perspective. Int J Nurs Stud 42:521-530, 2005

15. Caumo W, Schmidt AP, Schneider CN, Bergmann J, Iwamoto CW, Adamatti LC, Bandeira D, Ferreira MB: Risk factors for postoperative anxiety in adults. Anaesthesia 56: 720-728, 2001

16. Caumo W, Schmidt AP, Schneider CN, Bergmann J, Iwamoto CW, Adamatti LC, Bandeira D, Ferreira MBC: Preoperative predictors of moderate to intense acute postoperative pain in patients undergoing abdominal surgery. Acta Anaesthesiol Scand 46:1265-1271, 2002

17. Chamberlain K, Petrie K, Azariah R: The role of optimism and sense of coherence in predicting recovery following surgery. Psychology \& Health 7:301-310, 1992

18. Chan J, Ong J, Avalos G, Regan P, McCann J, Groarke A, Kelly J: Illness representations in patients with hand injury. J Plast Reconstr Aesthet Surg 62:927-932, 2009

19. Chang EC: Does dispositional optimism moderate the relation between perceived stress and psychological well-being?: A preliminary investigation. Pers Individ Dif 25:233-240, 1998

20. Charlson ME, Pompei $P$, Ales KL, MacKenzie CR: A new method of classifying prognostic comorbidity in longitudinal studies: Development and validation. J Chronic Dis 40: 373-383, 1987

21. Charlton JE: Acute and postoperative pain, in Charlton J (ed): Core Curriculum for Professional Education in Pain. Seattle, WA,, IASP Press, 2005, pp 125-138

22. Cleeland C, Ryan KM: Pain assessment: global use of the Brief Pain Inventory. Ann Acad Med Singapore 23:129-138, 1994

23. Cohen F, Kearney KA, Zegans LS, Kemeny ME, Neuhaus JM, Stites DP: Pessimism as a predictor of emotional morbidity one year following breast cancer surgery. PsychoOncology 13:309-320, 1999
24. Cole-King A, Harding KG: Psychological factors and delayed healing in chronic wounds. Psychosom Med 63: 216-220, 2001

25. Costello NL, Bragdon $E E$, Light $K C$, Sigurdsson $A$, Bunting S, Grewen K, Maixner W: Temporomandibular disorder and optimism: relationships to ischemic pain sensitivity and interleukin-6. Pain 100:99-110, 2002

26. Cousins MJ, Brennan F, Carr DB: Pain relief: a universal human right. Pain 112:1-4, 2004

27. Cronbach LJ: Coefficient alpha and the internal structure of tests. Psychometrika 16:297-334, 1951

28. De Groot $K$, Boeke S, Berge $H$, Duivenvoorden $H_{\text {, }}$ Bonkea B, Passchier J: The influence of psychological variables on postoperative anxiety and physical complaints in patients undergoing lumbar surgery. Pain 69:19-25, 1997

29. Dihle A, Helseth S, Paul SM, Miaskowski C: The exploration of the establishment of cutpoints to categorize the severity of acute postoperative pain. Clin J Pain 22: 617-624, 2006

30. Ebrahimpour PB, Do HT, Bornstein LJ, Westrich GH: Relationship between demographic variables and preoperative pain and disability in 5945 total joint arthroplasties at a single institution. J Arthroplasty 26:133-137, 2011

31. Edwards RR, Goble L, Kwan A, Kudel I, McGuire L, Heinberg L, Wigley F, Haythornthwaite J: Catastrophizing, pain, and social adjustment in scleroderma: Relationships with educational level. Clin J Pain 22:639-646, 2008

32. Ferreira VM, Sherman AM: The relationship of optimism, pain, and social support to well-being in older adults with osteoarthritis. Aging Ment Health 11:89-98, 2007

33. Gagliese L, Gauthier LR, Macpherson AK, Jovellanos M, Chan VWS: Correlates of postoperative pain and intravenous patient-controlled analgesia use in younger and older surgical patients. Pain Med 9:299-314, 2008

34. Geers AL, Wellman JA, Fowler SL, Helfer SG, France CR: Dispositional optimism predicts placebo analgesia. J Pain $11: 1165-1171,2010$

35. Geers AL, Wellman JA, Helfer SG, Fowler SL, France CR: Dispositional optimism and thoughts of well-being determine sensitivity to an experimental pain task. Ann Behav Med 36:304-313, 2008

36. Gijshers K, Nicholson F: Experimental pain thresholds influenced by sex of experimenter. Percept Mot Skills 101: 803-807, 2005

37. Giltay EJ, Geleijnse JM, Zitman FG, Hoekstra T, Schouten RG: Dispositional optimism and all-cause and cardiovascular mortality in a prospective cohort of elderly dutch men and women. Arch Gen Psychiatry 61:1126-1135, 2004

38. Good M, Stanton-Hicks M, Grass JA, Anderson GC, Choi C, Schoolmeesters LJ, Salman A: Relief of postoperative pain with jaw relaxation, music and their combination. Pain 81:163-172, 1999

39. Hager K, Machein U, Krieger S, Platt D, Seefried G, Bauer J: Interleukin-6 and selected plasma proteins in healthy persons of different ages. Neurobiol Aging 15: 771-772, 1994

40. Hagger MS, Orbell S: A meta-analytic review of the common-sense model of illness representations. Psychol Health 18:141-184, 2003
1444

1445

1446

1447

1448

1449

1450

1451

1452

1453

1454

1455

1456

1457

1458

1459

1460

1461

1462

1463

1464

1465

1466

1467

1468

1469

1470

1471

1472

1473

1474

1475

1476

1477

1478

1479

1480

1481

1482

1483

1484

1485

1486

1487

1488

1489

1490

1491

1492

1493

1494

1495

1496

1497

1498

1499

1500

1501

1502

1503

1504

1505

1506 
41. Hamel MB, Toth M, Legedza A, Rosen MP: Joint replacement surgery in elderly patients with severe osteoarthritis of the hip or knee. Arch Internl Med 168:1430-1440, 2008

42. Hartrick CT, Kovan JP, Shapiro S: The numeric rating scale for clinical pain measurement: a ratio measure? Pain Pract 3: 310-316, 2003

43. Hermele S, Olivo EL, Namerow P, Oz MC: Illness representations and psychological distress in patients undergoing coronary artery bypass graft surgery. Psychol Health Med 12:580-591, 2007

44. Ip HYV, Abrishami A, Peng PWH, Wong J, Chung F: Predictors of postoperative pain and analgesic consumption: $A$ qualitative systematic review. Anesthesiology 111:657-677, 2009

45. Janssen SA: Negative affect and sensitization to pain. Scand J Psychol 43:131-137, 2002

46. Jones CA, Voaklander DC, Suarez-Almazor ME: Determinants of function after total knee arthroplasty. Phys Ther 83: 696-706, 2003

47. Kalkman CJ, Visser K, Moen J, Bonsel GJ, Grobbee DE, Moons KGM: Preoperative prediction of severe postoperative pain. Pain 105:415-423, 2003

48. Katz J, Poleshuck EL, Andrus $\mathrm{CH}$, Hogan LA, Jung BF Kulick DI, Dworkin RH: Risk factors for acute pain and its persistence following breast cancer surgery. Pain 119: 16-25, 2005

49. Kiecolt-Glaser JK, Page GG, Marucha PT, MacCallum RC, Glaser R: Psychological influences on surgical recovery perspectives from psychoneuroimmunology. Am Psychol 53:1209-1218, 1998

50. Koivula M, Tarkka MT, Tarkka M, Laippala P, PaunonenIlmonen M: Fear and anxiety in patients at different time-points in the coronary artery bypass process. Int J Nurs Stud 39:811-822, 2002

51. Kubzansky LD, Martin LT, Buka SL: Early manifestations of personality and adult health: a life course perspective. Health Psychol 28:364-372, 2009

52. Kurtz S, Ong K, Lau E, Mowat F, Halpern M: Projections of primary and revision hip and knee arthroplasty in the United States from 2005 to 2030. J Bone Joint Surg Am 89: 780-785, 2007

53. Learmonth ID, Young C, Rorabeck C: The operation of the century: Total hip replacement. Lancet 370:1508-1519, 2007

54. Leventhal $H$, Benyamini $Y$, Brownlee $S$, Diefenbach $M$, Leventhal E, Patrick-Miller L, Robitaille C: Illness representations: theoretical foundations, in Petrie KJ, Weinman J (eds): Perceptions of Health And IIIness: Current Research and Applications. Amsterdam, NL, Harwood Academic, 1997, pp 19-45

55. Leventhal H, Nerenz DR, Steele DJ: Illness representations and coping with health threats, in Baum A (ed): Handbook of Psychology and Health, vol. IV: Social Psychological Aspects of Health. Hillsdale, NJ, Erlbaum, 1984, pp 219-252

56. Lingard EA, Riddle DL: Impact of psychological distress on pain and function following knee arthroplasty. J Bone Joint Surg 89:1161-1169, 2007

57. Llewellyn CD, McGurk M, Weinman J: Illness and treatment beliefs in head and neck cancer: Is Leventhal's common sense model a useful framework for determining changes in outcomes over time? J Psychosom Res 63:17-26, 2007
58. Losina E, Walensky RP, Kessler CL, Emrani PS, Reichmann WM, Wright EA, Holt HL, Solomon $\mathrm{DH}$, Yelin $\mathrm{E}$, Paltiel AD, Katz JN: Cost-effectiveness of total knee arthroplasty in the United States - patient risk and hospital volume. Arch Int Med 169:1113-1121, 2009

59. Mahler H, Kulik J: Optimism, pessimism and recovery from coronary bypass surgery: prediction of affect, pain and functional status. Psychol Health Med 5:347-358, 2000

60. Mccarthy SC, Lyons AC, Weinman J, Talbot R, Purnell D: Do expectations influence recovery from oral surgery? An illness representation approach. Psychol Health 18: 109-126, 2003

61. McWilliams LA, Goodwin RD, Cox BJ: Depression and anxiety associated with three pain conditions: Results from a nationally representative sample. Pain 111:77-83, 2004

62. Meissner W, Mescha $S$, Rothaug J, Zwacka $S$, Goettermann A, Ulrich K, Schleppers A: Quality improvement in postoperative pain management - results from the QUIPS Project. Dtsch Ärztebl Int 105:865-870, 2005

63. Melzack R: The McGill Pain Questionnaire: major properties and scoring methods. Pain 1:277-299, 1975

64. Mitchell MS, Harel W, Kan-Mitchell J, LeMay LG, Goedegebuure $P$, Huang XQ, Hofman F, Groshen S: Active specific immunotherapy of melanoma with allogeneic cell lysates. Rationale, results, and possible mechanisms of action. Ann N Y Acad Sci 690:153-166, 1993

65. Morrison RS, Magaziner J, McLaughlin MA, Orosz G, Silberzweig SB, Koval KJ, Siu AL: The impact of postoperative pain on outcomes following hip fracture. Pain 103:303-311, 2003

66. Morton DL, Watson A, El-Deredy W, Jones A: Reproducibility of placebo analgesia: Effect of dispositional optimism. Pain 146:194-198, 2009

67. Moss-Morris R, Humphrey $\mathrm{K}$, Johnson MH, Petrie KJ: Patients' perceptions of their pain condition across a multidisciplinary pain management program: do they change and if so does it matter? Clin J Pain 23:558-564, 2007

68. Moss-Morris R, Weinman J, Petrie KJ, Horne R, Cameron LD, Buick D: The Revised Illness Perception Questionnaire (IPQ-R). Psychol Health 17:1-16, 2002

69. MunafoMRStevensonAnxietyandsurgicarecoveryReinterpretingthditeraturePsychosomRes 1:589-5962001

70. Nickinson RS, Board TN, Kay PR: Post-operative anxiety and depression levels in orthopaedic surgery: A study of 56 patients undergoing hip or knee arthroplasty. J Eval Clin Pract 15:307-310, 2009

71. Nilsdotter A, Aurell $Y$, Siösteen A, Lohmander L, Roos H: Radiographic stage of osteoarthritis or sex of the patient does not predict one year outcome after total hip arthroplasty. Ann Rheum Dis 60:228-232, 2001

72. Orbell S, Johnston M, Rowley D, Espley A, Davey P: Cognitive representations of illness and functional and affective adjustment following surgery for osteoarthritis. Soc Sci Med 47:93-102, 1998

73. PapaioannouMSkapinakisPamigo\$Mavreas/BroumasG, PalgimesiA:Therolefatastrophizingrtheprediction fostoperativepainPairMedl 0:1452-14592009

74. Pellino TA, Gordon DB, Engelke ZK, Busse $K L$, Collins MA, Silver CE, Norcross NJ: Use of nonpharmacologic interventions for pain and anxiety after total hip and total knee arthroplasty. Orthop Nurs 24:182-190, 2005 
14 The Journal of Pain

1633

1634

1635

1636

1637

1638

1639

1640

1641

1642

1643

1644

1645

1646

1647

1648

1649

1650

1651

1652

1653

1654

1655

1656

1657

1658

1659

1660

1661

1662

1663

1664

1665

1666

1667

1668

1669

1670

1671

1672

1673

1674

1675

1676

1677

1678

1679

1680

1681

1682

1683

1684

1685

1686

1687

1688

1689

1690

1691

1692

1693

1694

1695
75. Perkins $\mathrm{F}$, Kehlet $\mathrm{H}$ : Chronic pain as an outcome of surgery - a review of predictive factors. Anesthesiology 93: $1123-1133,2000$

76. Peters M: Optimism as a resiliency for chronic pain. Eur J Pain 13(Suppl 1):S7, 2009

77. Peters ML, Sommer M, Rijke JM, Kessels F, Heineman E, Patijn J, Marcus MAE, Vlaeyen JWS, van Kleef M: Somatic and psychologic predictors of long-term unfavourable outcome after surgical intervention. Ann Surg 245: 487-494, 2007

78. Petrie KJ, Weinman J: Why illness perceptions matter. Clin Med 6:536-539, 2006

79. Pinto $P$, Mclntyre T, Almeida A, Araújo-Soares V: The mediating role of pain catastrophizing in the relationship between presurgical anxiety and acute postsurgical pain after hysterectomy. Pain 153:218-226, 2012

80. Pinto PR, Mclntyre T, Fonseca C, Almeida A, AraujoSoares V: Pre- and post-surgical factors that predict the provision of rescue analgesia following hysterectomy. Eur J Pain, 14 AUG 2012 [Epub ahead of print]

81. Pinto PR, Mclntyre T, Nogueira-SIlva C, Almeida A, AraujoSoares V: Risk factors for persistent post-surgical pain in women undergoing hysterectomy due to benign causes: a prospective predictive study. J Pain 13:1045-1057, 2012

82. Pool GJ, Schwegler AF, Theodore BR, Fuchs PN: Role of gender norms and group identification on hypothetical and experimental pain tolerance. Pain 129:122-129, 2007

83. Rasmussen HN, Scheier MF, Greenhouse JB: Optimism and physical health: a meta-analytic review. Ann Behav Med 37:239-256, 2009

84. Riley JL, Robinson ME: CSQ: five factors or fiction? Clin J Pain 13:156-162, 1997

85. Roth ML, Tripp DA, Harrison MH, Sullivan M, Carson P: Demographic and psychosocial predictors of acute perioperative pain for total knee arthroplasty. Pain Res Manag 12: 185-194, 2007

86. Roykulcharoen V, Good M: Systematic relaxation to relieve postoperative pain. J Adv Nurs 48:140-148, 2004

87. Scharloo $M$, de Jong RJ, Langeveld TP, Velzen-Verkaik E, Akker MM, Kaptein AA: Quality of life and illness perceptions in patients with recently diagnosed head and neck cancer. Head \& Neck 27:857-863, 2005

88. Scheier MF, Carver SC: Effects of optimism on psychological and physical wellbeing: Theoretical overview and empirical update. Cognit Ther Res 16:201-228, 1992

89. Scheier MF, Carver SC: On the power of positive thinking: the benefits of being optimistic. Curr Dir in Psychol Sci 2:26-30, 1993

90. Scheier MF, Carver CS, Bridges MW: Distinguishing optimism from neuroticism (and trait anxiety, self-mastery, and self-esteem): a reevaluation of the life orientation test. J Pers Soc Psychol 67:1063-1078, 1994

91. Scheier MF, Matthews KA, Owens JF, Schulz R, Bridges MW, Magovern GJ, Carver CS: Optimism and rehospitalization after coronary artery bypass graft surgery. Arch Int Med 159:829-835, 1999
92. Scheier MF, Weintraub JK, Carver CS: Coping with stress: Divergent strategies of optimists and pessimists. J Pers Soc Psychol 51:1257-1264, 1986

93. Schou I, Ekeberg $\varnothing$, Ruland CM, Sandvik I, Karesene R: Pessimism as a predictor of emotional morbidity one year following breast cancer surgery. Psycho-Oncology 13: 309-320, 2004

94. Schug SA, Pogatzki-Zahn EM: Chronic pain after surgery or injury. Pain-Clinical Updates 19:1-5, 2011

95. Seligman MEP: Learned optimism: How to change your mind and your life. New York, NY, Vintage Books, 2006

96. Sipila K, Ylöstalo PV, Ek E, Zitting P, Knuuttila ML: Association between optimism and self-reported facial pain. Acta Odontol Scand 64:177-182, 2006

97. Sjoling M, Nordahl G, Olofsson N, Asplund K: The impact of preoperative information on state anxiety, postoperative pain and satisfaction with pain management. Patient Educ Couns 51:169-176, 2003

98. Sniehotta FF, Gorski C, Araujo-Soares V: Adoption of community-based cardiac rehabilitation programs and physical activity following phase III cardiac rehabilitation in Scotland: A prospective and predictive study. Psychol Health 25:839-854, 2010

99. Stoddard JA, White KS, Covino NA, Strauss L: Impact of a brief intervention on patient anxiety prior to day surgery. J Clin Psychol Med Settings 12:99-110, 2005

100. Strassels SA, McNicol E, Suleman R: Postoperative pain management: A practical review, part 1. Am J Health-Syst Pharm 62:1904-1962, 2005

101. Sullivan M, Tanzer M, Reardon G, Amirault D, Dunbar M, Stanish W: The role of presurgical expectancies in predicting pain and function one year following total knee arthroplasty. Pain 152:2287-2293, 2011

102. Symreng I, Fishman S: Anxiety and pain. Pain: Clinical Updates XII:1-6, 2004

103. Taenzer $P$, Melzack R, Jeans ME: Influence of psychological factors on postoperative pain, mood and analgesic requirements. Pain 24:331-342, 1986

104. Ulmer JF: An exploratory study of pain, coping, and depressed mood following burn injury. J Pain Symptom Manage 13:148-157, 1997

105. Vilardo L, Shah M: Chronic pain after hip and knee replacement. Tech Reg Anesth Pain Manag 15:110-115, 2011

106. Wong WS, Fielding R: Quality of life and pain in Chinese lung cancer patients: Is optimism a moderator or mediator? Qual Life Res 16:53-63, 2007

107. Wu CL, Raja SN: Treatment of acute postoperative pain. Lancet 377:2215-2225, 2011

108. Wylde V, Hewlett S, Learmonth ID, Dieppe P: Persistent pain after joint replacement: Prevalence, sensory qualities, and postoperative determinants. Pain 152:566-572, 2011

109. Zigmond AS, Snaith RP: The hospital anxiety and depression scale. Acta Psychiatr Scand 67:361-370, 1983
1696

1697

1698

1699

1700

1701

1702

1703

1704

1705

1706

1707

1708

1709

1710

1711

1712

1713

1714

1715

1716

1717

1718

1719

1720

1721

1722

1723

1724

1725

1726

1727

1728

1729

1730

1731

1732

1733

1734

1735

1736

1737

1738

1739

1740

1741

1742

1743

1744

1745

1746

1747

1748

1749

1750

1751

1752

1753

1754

1755

1756

1757

1758 